\title{
Accounting and Management Research
}

Recibido:

junio 9 de 2021

Aceptado:

octubre 15 de 2021.

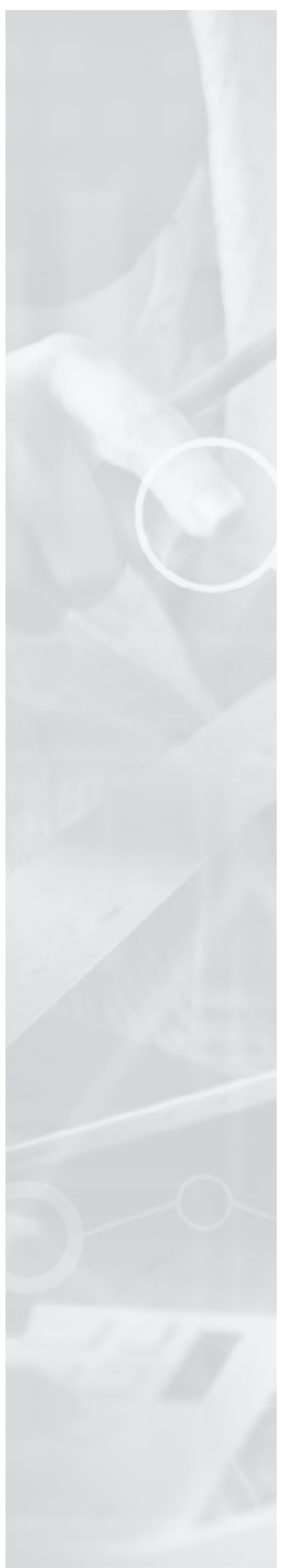

\section{Inteligencia emocional del contador público colombiano sancionado por la Junta Central de Contadores (JCC)}

\author{
Emotional intelligence of Colombian public accountants \\ sanctioned by the Central Board of Accountancy (JCC)
}

William A. Pirela Espina ${ }^{1}$

williampirela@gmail.com

ORCID ID: https://orcid.org/0000-0003-3811-222X

https://doi.org/10.22209/amr.v1n1a02

\section{Resumen}

La presente investigación tuvo como objetivo analizar el manejo de las herramientas de inteligencia emocional por parte de los profesionales contables al momento de enfrentar psicológicamente las sanciones impuestas por la Junta Central de Contadores Bogotá D.C., en Colombia desde el año 2016 hasta el año 2020. Este trabajo se enmarcó en las teorías de García et al. (2019), Enríquez et al. (2015), Hernández et al. (2014), entre otros. Metodológicamente, se presenta un estudio descriptivo, explicativo y de campo, con un muestreo discrecional, utilizándose para la recolección de datos la encuesta y el cuestionario basado en el Inventario del Cociente Emocional de BarOn (ICE BarOn); cuya confiabilidad se midió a través de la prueba Alfa de Cronbach con un coeficiente del 0,967. Los resultados demostraron que los contadores públicos encuestados han desarrollado habilidades para la regulación de sus emociones, demostrando un alto nivel de competencias intrapersonales (CIA) y estado de ánimo en general (CAG); sin embargo, tienen un promedio regular en el desarrollo de las competencias interpersonales (CIE), adaptabilidad (CAD) y manejo de emociones (CME). Se concluyó que los profesionales contables objeto de estudio, deben trabajar dimensiones intelectuales (conocimientos) y emocionales (afectivas) para desarrollar de forma integral su profesión y enfrentar de manera idónea las situaciones adversas.

1 Facultad de Ciencias Contables, Económicas y Administrativas. Universidad de Manizales, Caldas. Colombia 
Palabras claves: código de ética profesional; ejercicio profesional del contador público; manejo de emociones; procesos disciplinarios.

\begin{abstract}
This research study aimed to analyze how professional accountants manage emotional intelligence tools when psychologically coping with sanctions imposed by the Central Board of Accountancy (JCC), based in the Capital District of Bogotá (Colombia), from 2016 to 2020. This research study was framed in the theories of García et al. (2019), Enríquez et al. (2015), Hernández et al. (2014), among others. Methodologically, a descriptive, explanatory and field design was used with discrete sampling. Both an interview and a questionnaire based on the BarOn Emotional Quotient Inventory (BarOn EQ-i) were used as data collection instruments; The reliability coefficient of this instrument as measured through Cronbach's Alpha test was 0.967. The results showed that the surveyed public accountants have developed abilities to regulate their emotions, demonstrating a high level of intrapersonal competencies and general mood. However, the results were not particularly high in interpersonal competencies, adaptability and emotional management (CME). It was concluded that the accounting professionals under study must work on intellectual (knowledge) and emotional (affective) dimensions to comprehensively develop their profession and adequately face adverse situations.
\end{abstract}

Keywords: professional code of ethics; professional practice of the public accountant; managing emotions; disciplinary proceedings.

\title{
Introducción
}

El contador público es el profesional de las ciencias contables que está facultado por ley para dar fe pública sobre la información financiera de cualquier entidad y persona, por lo que se considera que el desempeño de sus actuaciones conlleva a un elevado nivel de riesgo económico y social. En consecuencia, Muñoz et al. (2020) exponen que los contadores públicos son profesionales que diariamente enfrentan situaciones en las cuales deben tomar decisiones desde el ámbito económico y financiero que, en muchos de los casos, les genera un constante estrés que afecta psicológicamente su bienestar, estando obligados a cumplir fielmente con los principios éticos inherentes al trabajo encomendado.

En Colombia, la UAE-Junta Central de Contadores Bogotá D.C., es el ente rector y regulador del ejercicio de la profesión del contador público, siendo a su vez 
responsable del control y vigilancia de cada una de las actuaciones a fin de asegurar el cumplimiento de las normas, reglas y la ética profesional, conforme con lo establecido en la Ley 43 de 1990, el Decreto 1510 de 1998, y la Resolución 42 de 1999 (García et al. 2019).

En ese sentido, afirma Ríos (2016) que algunos contadores públicos incumplen con las normas y principios en la realización de su trabajo, desconociendo las reglas de ética profesional, teniendo como consecuencia que la UAE-Junta Central de Contadores Bogotá D.C., sancione al profesional por los hechos ilícitos cometidos. Estas prácticas irregulares han ocasionado desconfianza en las labores realizadas por el profesional contable y su vez, ha empañado su imagen.

Por su parte, para Martínez y Franco (2014) los procedimientos disciplinarios aplicados por la UAE-Junta Central de Contadores Bogotá D.C., tales como amonestaciones, multas, suspensión y la cancelación de la inscripción profesional, garantizan el establecimiento de condiciones de seguridad jurídica a la comunidad en general, en cuanto a que las actuaciones del contador público están siendo supervisadas conforme con los lineamientos legales y los procedimientos establecidos en las normas reguladoras.

Por lo antes mencionado, el contador público debe generar y fortalecer habilidades para ser capaz de encontrar soluciones a eventualidades negativas que le permitan su desarrollo como persona y darle respuesta a cualquier inconveniente en su vida profesional, y poder manejar de forma adecuada situaciones adversas como la imposición de sanciones por conductas adoptadas al margen de las normas de ética profesional. Es por lo que, se ve en la necesidad de incurrir a la inteligencia emocional la cual sugiere el desarrollo individual de la autoconciencia, permitiendo identificar las emociones propias que se presenten en el momento, siendo capaz de regularlas, así como reconocer la de los demás.

En este contexto, el presente trabajo de investigación tuvo como objetivo analizar el manejo de las herramientas de inteligencia emocional por parte de los profesionales contables al momento de enfrentar psicológicamente las sanciones impuestas por la Junta Central de Contadores Bogotá D.C., en Colombia desde el año 2016 hasta el año 2020.

\section{Fundamentación teórica}

Actualmente, el manejo de las emociones en cualquier ámbito, se hace cada vez más importante, puesto que se controla el autoconocimiento y la autorregulación emocional; tomando en consideración que la inteligencia emocional no es 
más que una temática adoptada por la sociedad para el manejo de las habilidades y emociones interpersonales que se deben tener como base para un buen desarrollo profesional y personal. De acuerdo a Goleman (1998), la inteligencia emocional es la capacidad que tienen los individuos para reconocer los sentimientos propios y ajenos.

En el caso de la contaduría pública, se debe reconocer que se trata de una profesión ejercida por personas naturales, sensibles y susceptibles a cambios emocionales, ocasionados estos por una serie de factores que pueden influenciar el desempeño tanto laboral como profesional. Para Melendro (2018) la inteligencia emocional al ser aplicada por los contadores públicos que se enfrentan a alguna sanción dentro de su ejercicio profesional, le permitirá establecer relaciones personales tranquilas y exitosas, a través de una comunicación asertiva y el desarrollo de habilidades sociales que le favorecerán ante la situación en la que se encuentran.

Se considera a su vez que lo antes señalado es un factor importante dentro del desarrollo integral como profesionales, siendo necesario manejar adecuados niveles de inteligencia emocional para evitar los posibles daños en la salud física y psicológica que puedan generar las amonestaciones y sanciones que se presenten. La falta de regulación emocional tanto en contadores públicos como en cualquier otro campo profesional, trae consigo una serie de consecuencias negativas para el desarrollo de cotidianidad de cada persona.

En ese orden de ideas, el Estado colombiano al igual que en otros países, le asigna una cantidad de responsabilidades a los contadores públicos tipificadas en la ley, lo que hace que la profesión se vea enmarcada en grandes situaciones de riesgos, para lo cual se crea la Junta Central de Contadores a través del Decreto 2373 del 18 de septiembre de 1956 en la capital de dicho país, siendo esta la encargada de velar por el cumplimiento efectivo y eficaz de cada una de las atribuciones asignadas a los contadores; de lo contrario, está en la obligación de imponer una serie de sanciones y amonestaciones que regulen las acciones del profesional y el fiel cumplimiento de los principios establecidos.

Frente a esta situación, el profesional de la contaduría pública, se ve en la necesidad de acudir al dominio de la inteligencia emocional, siendo capaz de identificar y controlar sus emociones ante situaciones incomodas generadas por la entidad antes mencionada, asumiendo la responsabilidad de sus actos. 


\section{Materiales y métodos}

De acuerdo con Hernández et al. (2014) todo diseño investigativo se elabora con el propósito de dar respuesta a todas aquellas interrogantes de investigación planteadas y cumplir con los objetivos establecidos. El presente trabajo de investigación se enmarcó dentro de un estudio de tipo descriptivo, explicativo y de campo.

Para la creación de la base de datos que sirvió de fundamento al desarrollo del estudio, se obtuvo información cuantitativa publicada en el portal de la UAE-Junta Central de Contadores Bogotá D.C., a través del Registro de Sanciones Contadores de la Unidad Administrativa Especial - Junta Central de Contadores desde el año 1.992 hasta el 07-07-2021 que señala que han sido sancionados 1.357 contadores públicos.

En este sentido, las sanciones impuestas han sido clasificadas por la UAE-Junta Central de Contadores Bogotá D.C., de la siguiente forma:

- Amonestaciones: se considera la sanción menos grave y es impuesta cuando la falta cometida es leve.

- Multas: se aplica cuando la falta incurrida no se considera un delito o un incumplimiento grave de la ética profesional de acuerdo con lo establecido en el artículo 24 de la Ley 43 de 1990. La cuantía máxima es de cinco (5) salario mínimo mensual legal vigente, pudiendo ser sucesivas.

- Suspensión de la inscripción profesional: es impuesta cuando el profesional comete cualquiera de las conductas señaladas en el artículo 25 de la Ley 43 de 1990. Esta suspensión puede ser hasta de un (1) año.

- Cancelación de la inscripción profesional: es la pérdida definitiva de la tarjeta profesional del contador público y se considera la sanción más grave. Se aplica cuando se incurren en las conductas tipificadas en el artículo 26 de la Ley 43 de 1990. En el caso de sociedades de contadores públicos la cancelación puede ser aplicada por negligencia o dolo de la firma, cuando los socios o empleados no cumplen con las normativas profesionales o cuando el desarrollo del objeto se realiza incumpliendo con los requisitos señalados en la Ley 43 de 1990.

Para tal efecto, el estudio fue basado en un muestreo discrecional, conocido como muestreo intencional o de conveniencia en la selección de los contadores públicos sancionados que participaron en la investigación. Hernández et al. (2014) afirman que es una técnica de muestreo empleada para adquirir muestras con base a la facilidad de acceso a la información, disponibilidad de las personas que serán parte de la muestra e intervalo de tiempo específico. 
Para este trabajo de investigación se seleccionó una muestra de doscientos setenta y seis (276) contadores públicos sancionados por la UAE-Junta Central de Contadores Bogotá D.C., en el periodo comprendido desde el año 2016 hasta el año 2020 de acuerdo con el siguiente detalle:

Tabla 1. Cantidad de contadores públicos sancionados desde el año 2016 al año 2020

\begin{tabular}{|c|c|c|c|c|c|c|}
\hline \multirow{2}{*}{ Descripción } & \multicolumn{7}{|c|}{ Año } \\
\cline { 2 - 8 } & 2020 & 2019 & 2018 & 2017 & 2016 & Total \\
\hline Número de contadores sancionados & 17 & 26 & 19 & 87 & 127 & 276 \\
\hline
\end{tabular}

Fuente: elaboración propia, 2020.

Tabla 2. Detalle por género de los contadores públicos sancionados desde el año 2016 al año 2020

\begin{tabular}{|l|c|c|c|c|c|c|}
\hline \multirow{2}{*}{ Género } & \multicolumn{5}{|c|}{ Número de contadores sancionados } \\
\cline { 2 - 8 } & 2020 & 2019 & 2018 & 2017 & 2016 & Total \\
\hline Hombres & 8 & 18 & 12 & 55 & 58 & 151 \\
\hline Mujeres & 9 & 8 & 7 & 32 & 69 & 125 \\
\hline Total por Género & 17 & 26 & 19 & 87 & 127 & 276 \\
\hline
\end{tabular}

Fuente: elaboración propia, 2020.

Tabla 3. Tipo de sanciones impuestas a los contadores públicos desde el año 2016 al año 2020

\begin{tabular}{|l|c|c|c|c|c|c|}
\hline \multirow{2}{*}{ Tipo de sanción } & \multicolumn{6}{|c|}{ Número de contadores sancionados } \\
\cline { 2 - 8 } & 2020 & 2019 & 2018 & 2017 & 2016 & Total \\
\hline Amonestación & 1 & 0 & 2 & 5 & 10 & 18 \\
\hline Multas & 0 & 0 & 0 & 3 & 1 & 4 \\
\hline $\begin{array}{l}\text { Suspensión de la inscripción } \\
\text { profesional }\end{array}$ & 15 & 19 & 12 & 77 & 108 & 231 \\
\hline $\begin{array}{l}\text { Cancelación de la Inscripción } \\
\text { profesional }\end{array}$ & 1 & 7 & 5 & 2 & 8 & 23 \\
\hline Total tipo de sanciones & 17 & 26 & 19 & 87 & 127 & 276 \\
\hline
\end{tabular}

Fuente: elaboración propia, 2020. 
Cabe destacar que cada una de las tablas anteriores, fueron elaboradas a partir de la información suministrada por el Registro de Sanciones a Contadores de la Unidad Administrativa Especial-Junta Central de Contadores.

Por otra parte, de acuerdo a lo planteado por Sabino (2006) los instrumentos son todos aquellos medios materiales que se utilizan con el fin de adquirir y almacenar la información que se desea, entre los que se menciona: formatos de cuestionario, fichas, guías de entrevista, escala de actitudes o de opinión, entre otras. En ese sentido, se seleccionó como instrumento para la presente investigación, la encuesta; la cual es definida por Zapata (2006) como una técnica empleada para reunir de manera sistemática todos los datos que se desean recoger sobre determinado tema en relación a una población específica, a través de contactos directos con la persona o personas que lo integran.

A su vez, Arias (2006), establece que el cuestionario no es más que una modalidad de encuesta, la cual se implementa de forma escrita cumpliendo con un formato especifico contentivo de una serie de preguntas. Para la presente investigación, se diseñó un cuestionario compuesto por preguntas cerradas dado que se establecieron previamente las opciones de respuesta a ser elegidas por los encuestados.

Mestre y Fernández (2007), mencionan la existencia de tres instrumentos difundidos para medir la inteligencia emocional llamados:

- El Inventario del Cociente Emocional de BarOn de 1997.

- El Traid Meta-Mood Scale (TMMS) de Salovey y Mayer de 1990, y

- El Multifactor Emotional Intelligence (MSCEIT) Salovey y Mayer y Caruso de 2002.

Con base al contexto anterior, como instrumento para la recolección de datos se utilizó la encuesta que con la ayuda de un cuestionario basado en el Inventario del Cociente Emocional de BarOn (ICE BarOn), en el cual se midieron los quince (15) factores de la inteligencia personal, emocional y social a través de los siguientes componentes: 
Figura 1. Componentes del Cociente Emocional de BarOn.

\section{Intrapersonal (CIA):}

Incluye la comprensión emocional de sí mismo, asertividad, autoconcepto, autorrealización c independencia.

\section{Interpersonal (CIE):}

Considera la empatía, responsabilidad social y relación interpersonal.

\section{De adaptabilidad (CAD):}

Reúne prueba de la realidad, flexibilidad y solución de problemas

\section{Del manejo de emociones (CME):}

Incluye la tolerancia al estrés y control de los impulsos, y

\section{Del estado de ánimo en general (CAG):}

Evalúa la felicidad y el optimismo.

Fuente: elaboración propia, 2020

Dicho cuestionario fue enviado a través de la aplicación de Google Forms durante el primer trimestre del 2021 a los doscientos setenta y seis (276) contadores públicos sancionados por la UAE-Junta Central de Contadores Bogotá D.C., en el periodo comprendido desde el año 2016 hasta el año 2020. El cuestionario fue estructurado tipo Escala de Likert con cuatro opciones en las respuestas (1) muy rara vez, (2) rara vez, (3) a menudo y (4) muy a menudo.

En ese sentido, de la totalidad de cuestionarios enviados se recibieron doscientas siete (207) respuestas, que representó un setenta y cinco por ciento (75\%) de la muestra seleccionada para este trabajo de investigación. Una vez revisado y analizado el instrumento a través de cual se obtuvieron los datos, es necesario medirle su grado de fiabilidad o confiabilidad, lo que permite arrojar resultados reales. Al respecto, Hernández et al. (2014), establecen que la confiabilidad es el grado en el cual su aplicación repetida a un mismo sujeto conlleva a resultados iguales. 
Por consiguiente, la confiabilidad se basa en el grado a través del cual las mediciones de un instrumento son estables, reales, estables y libres de error. Dentro de ese marco, para la validez del instrumento de recolección de datos se utilizó la prueba Alfa de Cronbach que arrojó un resultado de 0,967 el cual se consideró un coeficiente significativamente alto (Hernández et al. 2014).

\section{Resultados}

Ahora bien, el instrumento aplicado en la investigación (cuestionario basado en el Inventario del Cociente Emocional de BarOn (ICE BarOn)) permitió recolectar los datos de la muestra no probabilística de doscientos siete (207) respuestas recibidas, obteniéndose los siguientes resultados:

Tabla 4. Componentes de la inteligencia emocional de los contadores públicos sancionados en el período comprendido entre el año 2016 y el año 2020.

\begin{tabular}{|l|c|c|c|c|c|}
\hline \multirow{2}{*}{\multicolumn{2}{|c|}{ Componente }} & \multicolumn{5}{c|}{ Respuestas recibidas } \\
\cline { 2 - 6 } & $\begin{array}{c}\text { Muy rara } \\
\text { vez }\end{array}$ & $\begin{array}{c}\text { Rara } \\
\text { vez }\end{array}$ & $\begin{array}{c}\text { A } \\
\text { menudo }\end{array}$ & $\begin{array}{c}\text { Muy a } \\
\text { menudo }\end{array}$ & Total \\
\hline Intrapersonal (CIA) & 14 & 21 & 41 & 131 & 207 \\
\hline Interpersonal (CIE) & 82 & 90 & 21 & 14 & 207 \\
\hline Adaptabilidad (CAD) & 22 & 78 & 71 & 36 & 207 \\
\hline Manejo de emociones (CME) & 21 & 76 & 69 & 41 & 207 \\
\hline $\begin{array}{l}\text { Estado de ánimo en general } \\
\text { (CAG) }\end{array}$ & 41 & 49 & 35 & 82 & 207 \\
\hline
\end{tabular}

Fuente: elaboración propia, 2020

Tabla 5. Componentes de la inteligencia emocional de los contadores públicos sancionados en el período comprendido entre el año 2016 y el año 2020 (Porcentaje de respuestas recibidas)

\begin{tabular}{|l|c|c|c|c|c|}
\hline \multirow{2}{*}{\multicolumn{1}{|c|}{ Componente }} & \multicolumn{5}{|c|}{ Respuestas recibidas (\%) } \\
\cline { 2 - 6 } & $\begin{array}{c}\text { Muy rara } \\
\text { vez }\end{array}$ & $\begin{array}{c}\text { Rara } \\
\text { vez }\end{array}$ & $\begin{array}{c}\text { A } \\
\text { menudo }\end{array}$ & $\begin{array}{c}\text { Muy a } \\
\text { menudo }\end{array}$ & Total \\
\hline Intrapersonal (CIA) & 6,76 & 10,14 & 19,81 & 63,29 & 100 \\
\hline Interpersonal (CIE) & 39,61 & 43,48 & 10,15 & 6,76 & 100 \\
\hline Adaptabilidad (CAD) & 10,63 & 37,69 & 34,30 & 17,39 & 100 \\
\hline Manejo de emociones (CME) & 10,14 & 36,72 & 33,33 & 19,81 & 100 \\
\hline $\begin{array}{l}\text { Estado de ánimo en general } \\
\text { (CAG) }\end{array}$ & 19,81 & 23,67 & 16,91 & 39,61 & 100 \\
\hline
\end{tabular}

Fuente: elaboración propia, 2020 
Por otra parte, el ser humano se divide en dos grandes grupos de acuerdo con el género, razón por la cual es importante resaltar en esta investigación que el Código de Ética Profesional establecido en la Ley 43 de 1990 en Colombia ha sido vulnerado desde el año 2016 hasta el año 2020 por 276 contadores públicos donde se encontró que el 54,71\% (151 contadores públicos) son del género masculino y 45,29\%(125 contadores públicos) del género femenino; es decir un 9,42\% (26 contadores públicos) mayor.

De acuerdo con la información publicada en el portal de la UAE-Junta Central de Contadores Bogotá D.C., a través del Registro de Sanciones Contadores de la Unidad Administrativa Especial - Junta Central de Contadores, durante el período comprendido entre el año 2016 hasta el año 2020, las sanciones aplicadas fueron:

- 231 suspensiones de la inscripción profesional (83,7\% del total de las sanciones aplicadas),

- 23 cancelaciones de la inscripción profesional (8,33\% del total de las sanciones aplicadas),

- 18 amonestaciones (6,52\% del total de las sanciones aplicadas), y

- 4 multas (1,45\% del total de las sanciones aplicadas).

A continuación se presentan los resultados de las respuestas recibidas sobre los componentes de la inteligencia emocional medidos con la aplicación del cuestionario basado en el Inventario del Cociente Emocional de BarOn (ICE BarOn):

- Componente Intrapersonal (CIA): la mayor frecuencia se presentó en la categoría muy a menudo con 131 respuestas que representó un 63,29 \% de las respuestas recibidas.

- Componente Interpersonal (CIE): la mayor incidencia fue en la opción rara vez con 90 respuestas que representa un 43,48 \% de las respuestas recibidas.

- Componente de Adaptabilidad (CAD): la opción más frecuente fue rara vez con 78 respuestas que representa un 37,69 \% de las respuestas recibidas.

- Componente del manejo de emociones (CME): obtuvo mayor frecuencia rara vez con 76 respuestas que representa un 36,72\% de las respuestas recibidas.

- Componente del estado de ánimo en general (CAG): la mayor incidencia fue en la opción muy a menudo con 82 respuestas que representa un $39,61 \%$ de las respuestas recibidas. 


\section{Discusión}

El trabajo de investigación fue realizado con el propósito de demostrar cómo los contadores públicos a través de las herramientas de la inteligencia emocional manejaron psicológicamente las sanciones impuestas por la UAE-Junta Central de Contadores Bogotá D.C., en Colombia desde el año 2016 hasta el año 2020.

En el análisis de los cinco (5) principales componentes conceptuales de la inteligencia emocional que incluyen los quince (15) factores medidos por el cuestionario basado en el Inventario del Cociente Emocional de BarOn (ICE BarOn) los resultados demostraron que los contadores encuestados en muchas ocasiones tuvieron una comprensión emocional de sí mismo, siendo asertivos en el manejo de las situaciones presentadas como consecuencia de las sanciones impuestas, lo cual se sustenta con las respuestas recibidas, por cuanto la gran mayoría seleccionó la opción "Muy a menudo" ante las siguientes frases presentadas en la encuesta:

- Sé cómo enfrentarme a aquellos problemas desagradables.

- Cuando enfrento una situación difícil acostumbro reunir toda la información que pueda sobre ella.

- Considero que puedo controlarme ante situaciones que se tornen muy difíciles.

- Cuando quiero resolver un problema analizo cada una de las posibles soluciones y luego elijo la que considero mejor.

En tal sentido, los contadores públicos encuestados demostraron a través de sus respuestas que siempre se han enfocado en el desarrollo de competencias en áreas tradicionales de su profesión, dejando a un lado el fortalecimiento de herramientas cognitivas y emocionales que le ayuden a conocer aquellas actividades que pueden estar incumpliendo las reglas de la ética profesional que afecta de forma negativa la profesión, lo que coincide con lo afirmado por Salinas (2020) con relación a la necesidad de fortalecer aspectos relacionados a los sentimientos, sin descuidar el conocimiento profesional.

En ese orden de ideas, García (2020) consideró que la inteligencia emocional complementa los conocimientos teóricos y científicos del individuo que le ayudarán a controlar las emociones y reacciones proactivas para enfrentar los retos profesionales y personales.

Es preciso aseverar, que de acuerdo con las respuestas recibidas de los contadores públicos sancionados, en pocas ocasiones tuvieron empatía de la situación presentada desconociendo en algunas ocasiones la responsabilidad ante la comunidad, es decir que casi nunca comprendieron la solución del problema 
sucedido, lo que se manifestó en un lenguaje poco apropiado que les permitiera expresar sus emociones acordes con el nivel profesional. Tal resultado coincide con la opinión de Faltas (2017) quien afirmó que la inteligencia emocional es la posibilidad que los individuos tienen para el uso de la información que le brindan las emociones al momento de tomar decisiones y solucionar los problemas que se presente en su entorno.

Por consiguiente, de acuerdo con lo respondido por los contadores públicos en muy pocas ocasiones manejaron y controlaron el estrés y los impulsos generados por las sanciones, lo que les dificultó el trabajo bajo presión, percibiendo la situación real de las acciones cometidos y que originaron las sanciones recibidas. En este sentido, Jiménez (2018) sostuvo que la inteligencia emocional forma parte de la inteligencia social que le permite al individuo controlar las emociones y sentimientos para diferencias entre ellos y usar la información para guiar las acciones y pensamiento de las personas.

De igual manera, los contadores públicos encuestados desarrollaron diversas actividades que le generaron cambios en sus emociones, las cuales fue influyentes en su desempeño profesional, laboral y personal, por lo que se hace necesario que identifique la importancia del manejo de la inteligencia emocional en la realización de las actividades de su profesión para identificar los factores que influyen de forma negativa que incida en el manejo inadecuado de sus emociones.

Este resultado coincide con lo afirmado por Goleman (2014) quien definió la inteligencia emocional como la capacidad del individuo para automotivarse, insistir en acciones para lograr el objetivo planteado sin considerar los efectos negativos y las frustraciones, controlar los impulsos y evitar que las situaciones angustiantes interfieran con la racionabilidad del individuo.

De tal modo, que uno de los principales factores que influyó en la inteligencia emocional de los contadores públicos encuestados estuvo representado por la constante supervisión y presión realizadas por los organismo reguladores de su actuación, en especial la UAE-Junta Central de Contadores Bogotá D.C., lo que corrobora lo señalado por Franco (2017) quien consideró que la inteligencia emocional se relaciona con la inteligencia social que influye en la habilidad y capacidad de los individuos para adaptarse espontáneamente a las circunstancias del medio social y laboral.

Por otra parte, en el contexto empresarial, un manejo inadecuado de la inteligencia emocional de los contadores públicos encuestados también ejerció negativamente en las decisiones tomadas por estos, por cuanto no fueron capaces de comprender las causas que le generaron estrés y que influyeron en las relaciones 
profesionales con sus colegas. Al respecto, Luy (2019) señaló que la inteligencia emocional está representada por la capacidad que le permite a cada persona hacer uso de sus emociones y lograr actuar adecuadamente con el medio ayudándolo a adaptarse y relacionarse de forma saludable.

Así pues, los contadores públicos encuestados que demostraron un bajo nivel de inteligencia emocional. Tuvieron mayor dificultad para establecer relaciones con otros colegas y manejar el estrés originando agotamiento y conflictos en los equipos de trabajo, respondiendo "Muy rara vez" a la frase planteada "Soy capaz de respetar a los demás". Este resultado corrobora lo afirmado por Enríquez et al. (2015) quienes consideran que la inteligencia emocional le permite al individuo autoconocerse, entender el comportamiento de los otros y equilibrar las emociones orientados con la productividad, el rendimiento y la realización personal.

\section{Conclusiones}

El nivel de inteligencia emocional manejado por los contadores públicos sancionados les ha permitido afrontar de manera asertiva las situaciones de estrés generadas, ayudándolos a tomar decisiones acertadas para enfrentar las consecuencias de las conductas de incumplimiento a las normas profesionales y de ética que conllevaron su aplicación. Por consiguiente, el desarrollo de un nivel elevado de inteligencia emocional en los profesionales de la contaduría pública en el marco de las organizaciones ayudó a incrementar la calidad del trabajo en equipo y la capacidad para la adaptación a los cambios.

En este sentido, la inteligencia emocional le permitió al contador público sancionado por la UAE-Junta Central de Contadores Bogotá D.C., ser más asertivo y lograr relaciones emocionales mucho más sanas con el resto de los profesionales, contribuyendo a la generación de un buen nivel de sinergia en los equipos de trabajo y ser más comprometidos y productivos en la realización de sus actividades.

Es de vital importancia para los profesionales contables, desarrollar herramientas de inteligencia emocional para tener la posibilidad de adaptarse a situaciones relacionadas con el trato entre los profesionales y sus clientes, así como a la posibilidad de realizar tareas donde la empatía y el trabajo en equipo sean esenciales. En definitiva, quedó demostrado que las emociones del contador público encuestado jugaron un papel importante en la forma de reaccionar ante las situaciones adversas presentadas resultando imposible desvincular los sentimientos con las conductas asumidas. 
Finalmente, se hace necesario considerar que la formación técnica del profesional sea complementada con un fortalecimiento de su inteligencia emocional con el propósito de lograr metas mucho más altas de las planteadas al tener equilibrio en el manejo de las sentimientos y reacciones ante situaciones adversas como puede considerarse la aplicación de sanciones por parte de la UAE-Junta Central de Contadores Bogotá D.C., desde el año 2016 hasta el año 2020 y que constituye un aspecto fundamental para el desarrollo integral del contador público y el logro de su éxito profesional. En todo caso, lo antes señalado deberá estar articulado al componente ético que debe propenderse durante el ejercicio de la profesión de la contaduría pública, toda vez que, si las acciones del contador público se ajustan a la norma vigente y a los principios éticos, se evitarían cualquier acción disciplinaria que a su vez pueda afectar su desarrollo personal.

\section{Bibliografía}

Arias, F. (2006). El Proyecto de Investigación Científica. Editorial Episteme.

Enríquez, E.; Martínez, J. y Guevara, L. (2015). Relación de la inteligencia emocional con el desempeño laboral. Revista Ciencia \& Salud.

Faltas, I. (2017). Tres Modelos de Inteligencia Emocional. Dialnet, 1-3. https:// www.researchgate.net/publication/314283649_Tres_Modelos_de _Inteligencia_Emocional

Franco, J. (2017). Inteligencia Emocional y Bienestar Laboral de los Servidores Públicos del Consejo Nacional para la Igualdad Intergeneracional (tesis de posgrado). Universidad Central del Ecuador, Quito- Ecuador.

García, C. (2020). La inteligencia emocional en el desarrollo de la trayectoria académica del universitario. Revista Cubana de Educación Superior.

García, D.; Perilla, M. y Rojas, P. (2019). Comportamientos que conllevan sanciones de los Contadores Públicos por suspensión en el periodo 2016 al 2018. Repositorio Universitaria Agustiniana UniARi. http://repositorio.uniagustinia na.edu.co// handle/123456789/1140

Goleman, D. (2014). Inteligencia emocional. Kairós.

Goleman, D. (1998). The emotional intelligence of leaders. https://doi.org/10.1002/ |t|.40619981008

Hernández, R., Fernández, C. y Baptista, P. (2014). Metodología de la Investigación. Bogotá: McGraw Hill.

Jiménez, A. (2018). Inteligencia emocional. https://www.aepap.org/sites/default/files/ 457-469_inteligencia_emocional.Pdf

Luy, C. (2019). El aprendizaje basado en problemas (ABP) en el desarrollo de la inteligencia emocional de estudiantes universitarios. Propósitos y Representaciones.

Martínez, E. y Franco, R. (2014). El proceso disciplinario aplicado a los contadores públicos. Revista Libre Empresa. 
Melendro, C. (2018). Inteligencia Emocional y Liderazgo del Contador Público de UNIMINUTO (Trabajo de Grado). Corporación Universitaria Minuto de Dios. Bogotá D.C. Colombia.

Mestre, J., y Fernández, P. (2007). Manual de inteligencia emocional. https://dialnet. unirioja.es/servlet/libro?codigo $=275716$

Muñoz, J.; Castillejo, S. y Sarchi, M. (2020). Infracciones a la ética profesional y sanciones impuestas a los contadores públicos 2015-2019. Bogotá: Fundación Universitaria del Área Andina. https://digitk.areandina.edu.co/bitstream/handle/areandina/3788/ Trabajo\%20de\%20grado.pdf?sequence=4\&isAllowed=y

Ríos, J. (2016). Casos de infracción de los contadores públicos al código de ética entre los años 2011-2015 un acercamiento desde las sanciones de la Junta Central de Contadores. https://ciencia.lasalle. edu.co/contaduria_publica/613

Sabino, C. (2006). ¿Cómo hacer una tesis y elaborar toda clase de trabajos escritos? Editorial Panamericana.

Salinas-González, N. (2020). Manejo de la inteligencia emocional en docentes universitarios. Revista UniNorte de Medicina y Ciencias de la Salud.

Zapata, O. (2006). Metodología para llevar a cabo una investigación. Editorial Mc Graw Hill. Interamericana de México, D.F. 